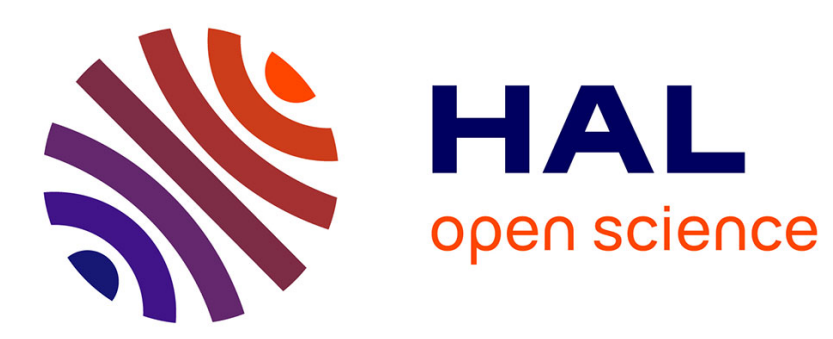

\title{
Vie professionnelle et maternité : représentations comparées de jeunes entrepreneures et cadres en TPE et PME en croissance
}

\author{
Marina Bourgain, Pierre Chaudat
}

\section{- To cite this version:}

Marina Bourgain, Pierre Chaudat. Vie professionnelle et maternité : représentations comparées de jeunes entrepreneures et cadres en TPE et PME en croissance. Revue de l'Entrepreneuriat, 2015, 14 (2), 10.3917/entre.142.0083 . hal-01745466

\section{HAL Id: hal-01745466 https://univ-lyon3.hal.science/hal-01745466}

Submitted on 21 Nov 2019

HAL is a multi-disciplinary open access archive for the deposit and dissemination of scientific research documents, whether they are published or not. The documents may come from teaching and research institutions in France or abroad, or from public or private research centers.
L'archive ouverte pluridisciplinaire HAL, est destinée au dépôt et à la diffusion de documents scientifiques de niveau recherche, publiés ou non, émanant des établissements d'enseignement et de recherche français ou étrangers, des laboratoires publics ou privés. 


\title{
Vie professionnelle et maternité : représentations comparées de jeunes entrepreneures et cadres en TPE et PME en croissance
}

\author{
Marina Bourgain et Pierre Chaudat \\ Marina Bourgain : Groupe ESC-Clermont, 4, Bd Trudaine F-63037 Clermont-Ferrand \\ CleRMa ; Chercheur associée Cereq ; Chercheur associée Magellan
}

Pierre Chaudat: Ecole Universitaire de Management IAE de Clermont, 26, Av. Léon Blum F-63000 Clermont-Ferrand

Marina BOURGAIN est Enseignante-Chercheure en Sciences de Gestion au Groupe ESC Clermont, membre du CRCGM (EA-3849), de l'Equipe associée Cereq-Clermont et de la Chaire Capital Humain de l'Université de Bordeaux. Elle a été Lecturer invitée à UC-Santa Cruz et Chercheur invitée à la Stanford Law School. Ses domaines de recherche portent sur les cadres en PME, la GRH et l'impact de l'Europe sur les pratiques de restructuration.

Pierre CHAUDAT est Maître de Conférences HDR en Sciences de Gestion à l'Ecole Universitaire de Management / IAE de Clermont-Ferrand I. Il est membre du CRCGM : Centre de Recherche Clermontois en Gestion et Management (EA-3849). Ses domaines de recherche portent essentiellement sur la santé au travail et la protection sociale des travailleurs indépendants. Pendant 8 ans, il a été chargé d'études et de missions au Régime Social Indépendant du Languedoc-Roussillon. Il est l'auteur de plusieurs articles dans des revues scientifiques et professionnelles.

\section{Mots clés en français et en anglais}

Entrepreneure / Femme Cadre / Maternité / PME en croissance /Mampreneur Women Entrepreneurs / Women professionals / Maternity / Growth SME / Mumpreneur

\section{Résumé}

Les récents débats sur les liens travail-famille et sur l'usage des dispositifs légaux des congés parentaux mettent en perspective les représentations comparées de la vie professionnelle et de la maternité de vingtquatre entrepreneures en TPE et cadres de PME en croissance. Le discours des mampreneurs souligne l'entreprise comme une opportunité de conciliation travail-famille ; alors que celui des cadres témoigne d'un ethos où la maternité est souvent perçue comme un frein professionnel. Notre apport principal consiste en la prise en compte de d'éléments de contexte comme le statut dans la modélisation des liens travail-famille.

\footnotetext{
Abstract

Recent debates on the work/family links within the French legal context shed a new light on the discourses of 24 entrepreneurs and executives in growth-oriented SMEs about their representation of career and of motherhood. The entrepreneurs discourse presents the company as a real opportunity to balance work and family; while the executives' discourse is embedded in a professional ethos whereby motherhood is often perceived as a career barrier. Our main contribution consists in taking contextual elements into account when modeling the connections between work and family.
} 


\section{Vie professionnelle et maternité : représentations comparées de jeunes entrepreneures et cadres en TPE et PME en croissance}

La stratégie Europe 2020 pour une croissance « intelligente, durable et inclusive » vise à relancer la croissance et le développement durable à travers un taux d'emploi de plus de $75 \%$. Pour atteindre cet objectif, le taux d'emploi des femmes ${ }^{1}$ doit dépasser les $60 \%$, ce qui implique de nouveaux défis pour articuler les sphères privée et professionnelle suivant un mode cumulatif. Un bouleversement des organisations économiques et sociales des pays industrialisés est donc à l'œuvre, lié à l'entrée en masse des femmes dans l'enseignement supérieur et dans le salariat notamment dans les secteurs du tertiaire, du commerce ou du service à la personne (Esping-Andersen, 2009 ; Goldin, 2006 ; Chauvel, 2010).

En France, les femmes représentent, en 2010, près de $48 \%$ de la population active (Banque Publique d'Investissement BPI, 2014 :3). Parmi les cadres du secteur privé, les femmes restent cependant minoritaires (34\% selon l'APEC, 2011), de même que parmi les entrepreneurs (33\% selon Blanchard, 2009), malgré une forte progression au cours des dix dernières années.

L'idée communément partagée est que le fait d'être chef d'entreprise ou cadre est particulièrement exigeant en termes d'investissements subjectif, émotionnel et pratique dans toute entreprise en croissance. Si la littérature sur le thème de l'articulation vie professionnellevie familiale s'est considérablement développée (Pailhé et Solaz, 2009 ; Silvera, 2002) dans un contexte de promotion de l'égalité des sexes tant dans la vie familiale que professionnelle (Martin, 2010), on note que les études empiriques restent cantonnées dans les grandes entreprises ou le secteur public (Fusulier, 2011a et 2007; Saint-Onge, Renaud, Guérin et Caussignac, 2002). Cette thématique a été peu explorée dans le champ des PME (voir notre revue de littérature). A ce sujet, de nombreux auteurs nous mettent en garde contre l'idée de concevoir les petites entreprises comme des modèles réduits de la grande entreprise (Torres, 2007). Par ailleurs, il existe un intérêt croissant pour l'entrepreneuriat des femmes. Les liens travail-famille des entrepreneurs ont notamment fait l'objet de travaux empiriques anglo-saxons (Schindehutte, Morris et Brennan., 2003; Loscocco et Leicht, 1993 ; Parasuraman, Purohit, Godshalk et Beutell, 1996 ; Fitzgerald et Winter, 2001). Néanmoins, les résultats de ces études ne sont pas a priori généralisables en France. En effet, les liens « travail-famille » sont socialement construits à travers les politiques publiques, et la législation du travail et la division entre travail rémunéré et non-rémunéré sont différentes selon les pays.

\footnotetext{
${ }^{1}$ Brewster et Rindfuss (2000), montrent sur près de 40 pays développés que les taux de fécondité et d'emploi sont positivement corrélés depuis 1990 (alors qu'ils l'étaient négativement dans les années 70). Données 2013, sources World Population Prospects, INED, EUROSTAT, par Pays = [taux de Fécondité] (taux d'activité). Etats-Unis=[2,06] (72,5\%); France $=[2,04](66 \%) ; \quad$ Ireland $=[2,05](62 \%)$; Angleterre $=[1,97](70 \%)$; Suède $=[1,9](78 \%) ;$ Italie $=[1,4](51 \%)$. Les pays méditerranéens ont des taux de fécondité et d'activité des femmes parmi les plus bas.
} 
Les plus récentes recherches sur l'entrepreneuriat féminin abordent les expériences des femmes, non plus en comparaison avec les hommes, mais à travers des recherches qualitatives portant sur les femmes dans leur multiplicité (Carrier, Julien et Menvielle, 2006 ; Cornet et Constantinidis, 2004). C'est dans cette même perspective que notre étude porte sur les représentations et les usages des congés légaux pour raisons parentales chez les femmes entrepreneurs et cadres en PME en croissance. Nous cherchons ainsi à comprendre leur attitude vis-à-vis de l'entreprise, présentée ou non comme une opportunité de concilier maternité et vie professionnelle. Pour ce faire, nous nous penchons sur les questions suivantes : connaissentelles leurs droits et quels usages disent-elles en faire ? La maternité est-elle considérée comme un frein, un levier ou une mise en veilleuse de leurs objectifs professionnels ? Quel rôle attribuent-elles à leur entourage ? Notre étude a pour objectif d'affiner et de contextualiser les représentations des liens travail-famille, à travers notamment la variable du statut des femmes (dirigeante, cadre), une variable peu investiguée.

Nous exposons dans une première partie une revue des travaux sur les liens travailfamille et sur les dispositions légales des congés parentaux en France. La deuxième partie détaille les aspects méthodologiques de la recherche, notamment la constitution de nos échantillons de cadres et de dirigeantes. Puis, les résultats de notre recherche exploratoire sont discutés dans la troisième partie.

\section{Revue des travaux sur les liens travail-famille et les dispositifs légaux du congé maternité et du congé parental salarié en France.}

Il est nécessaire de rappeler (1.1) les trois perspectives majeures de la recherche sur les liens travail-famille en présentant succinctement les théories, les concepts et les résultats sousjacents, avant de développer (1.2) les dispositions légales en vigueur en France liées aux congés parentaux.

\subsection{Perspectives majeures sur les liens travail-famille}

La relation travail-famille est une problématique sociale majeure pour les institutions, entreprises et Etats (Kanter, 1977). La recherche a suivi trois perspectives. La perspective dite «négative »s'attache aux conflits entre les sphères travail et famille avec un sens de la relation unidirectionnelle ou bidirectionnelle. La perspective dite «positive»s'intéresse aux bénéfices mutuels entre les deux sphères avec des relations multidirectionnelles. Enfin, la perspective d'intégration se penche sur la coévolution des deux sphères. Chaque perspective mobilise des concepts différents.

Les premières études ont ainsi porté sur les difficultés à articuler la sphère privée avec celle du travail, à travers les concepts de conflit, de tension et de compétition (Jennings et McDougald, 2007 ; Greenhaus et Beutell, 1985). Parmi les théories sous-jacentes à ce courant, la théorie néoclassique de la rareté des ressources (comme le temps et l'énergie) ainsi que la théorie des conflits postulent qu'un rôle assumé dans une sphère influe négativement sur le rôle dans l'autre sphère (Saint-Onge, Renaud, Guérin et Caussignac, 2002). La théorie instrumentale avec le travail comme obligation et la théorie des débordements "négatifs » ont les mêmes implications, l'insatisfaction dans une sphère affectant l'autre sphère (Sumer et Knight, 2001 ; Rothbard, 2001). Ces recherches ont tenté d'établir l'importance, le sens et les déterminants des conflits et des principales causes de stress dans l'emploi et la famille. Certains mécanismes de réduction du conflit travail-famille ont été explorés : outre l'importance du soutien concret du 
conjoint (Nikina, Le Loarne et Shelton, 2012), on signale l'environnement de travail et des caractéristiques de l'emploi (Saint-Onge, 2002), voire un revenu familial élevé (Chenevier, 1996), l'appui des dirigeants, des supérieurs et des collègues et les dispositions offertes, par les employeurs (Tremblay et Larrivière, 2009).

A l'inverse, la perspective dite "positive » postule que les rôles liés au travail et à la famille peuvent être bénéfiques l'un pour l'autre. Plusieurs concepts lui sont attachés: la facilitation, la compensation, le débordement positif, l'enrichissement et la congruence. Le concept de «facilitation» (Barnett et Rivers, 1998; Barnett et Hyde, 2001) postule que la multiplicité de rôles « de qualité » est bénéfique en termes de possibilités de réussite (meilleure estime de soi, défi, stimulation) et de supports pour l'individu (opportunités de relations sociales, ressources financières) - sachant que le travail rémunéré est généralement plus gratifiant que le travail familial. La compensation postule une sorte de régénération: l'insatisfaction (ou le stress) dans une sphère pousserait l'individu à rechercher satisfactions et récompenses dans l'autre sphère. Le concept de positive spillover (Rothbard, 2001 ; Grzywacz et Marks, 2000) fait référence aux débordements positifs d'une sphère sur l'autre en matière d'émotions, de valeurs, d'habilités comme la résolution de problèmes. Le concept d'enrichissement (Greenhaus et Powell, 2006) exprime le fait que l'interface " emploi-famille » peut être à l'origine du développement de compétences utiles dans l'exercice d'un rôle.

Les deux perspectives précédentes présentent les sphères travail et famille comme séparées; la troisième perspective s'intéresse à leur coévolution. Ainsi Barrère-Maurisson (1992, 2003) a montré que les carrières très promotionnelles sont associées à des formes familiales précises : homme marié dont la femme ne travaille pas, femme célibataire, ou, lorsque les deux travaillent, l'une des carrières se fait au détriment de l'autre. De même, la répartition du travail non-rémunéré est d'autant plus égalitaire que la catégorie professionnelle des deux conjoints est identique et élevée, et la «paternité active » est favorisée lorsque les deux conjoints sont attachés à leur carrière (Méda, 2009 ; Boyer, 2004). Selon cette perspective, le lien travail-famille est une construction sociale qui dépend du type de division du travail prévalant entre hommes et femmes. Ce lien évolue donc et se transforme au gré des changements de valeurs, des pratiques de gestion et des lois ainsi que des transformations économiques.

\section{Les pratiques de conciliation emploi-famille dans les petites structures.}

Seules deux études quantitatives anglo-saxonnes ont intégré des PME dans leur étude sur la conciliation emploi-famille. Leurs questions de recherche et leurs échantillons sont différents et leurs résultats ne convergent pas. Selon l'enquête Business Work-Life-Study (BWLS), menée aux Etats-Unis auprès d'un échantillon représentatif de 1057 entreprises de plus de 100 salariés (84\% secteur privé ; $16 \%$ secteur public et associatif), la présence de dispositifs de conciliation est liée à trois facteurs: le secteur d'activité, la taille de l'organisation, enfin une proportion égale de femmes et d'hommes cadres supérieurs. Ainsi, plus l'entreprise est grande, plus elle offre une gamme large de dispositifs. A taille équivalente, l'assurance, l'immobilier et la banque développent plus ces dispositifs que les entreprises de commerce (Families and Work Institute, 1998). Quant à l'enquête du Comité Intersectoriel du Secteur Privé au Québec (CISP, citée par Tremblay 2012) auprès de 200 établissements (80\% d'établissements de moins de 250 salariés, dont $44 \%$ filiales de groupes plus importants), elle ne s'intéressait qu'à l'usage de mesures de conciliation par les salariés. Selon l'Enquête CISP, les établissements de petite taille permettent une meilleure articulation travail-famille (généralement de manière informelle) que les grands établissements car nombre de petits 
établissements offrent des contextes plus facilitants, même si cela conduit parfois à de l'arbitraire (Tremblay, 2012: 234). Les horaires flexibles font partie des mesures les plus diffusées (17\% des établissements). Les mesures les moins diffusées sont le cheminement de carrière adapté aux exigences familiales et le travail à domicile (respectivement $3 \%$ et $5 \%$ des établissements). Par ailleurs, contrairement à BWLS, l'enquête CISP indique que l'usage de mesures de conciliation n'est pas lié à des secteurs d'activité particuliers ou à un taux de féminisation élevé de l'encadrement. En revanche, cette même enquête souligne le lien avec la stratégie globale de l'organisation : lorsque la stratégie est orientée vers la réduction des coûts, l'articulation travail-famille est peu en usage. A l'inverse, une stratégie d'innovation induit une culture d'entreprise favorisant l'usage de mesures d'articulation travail-famille. Deux hypothèses pourraient, selon nous, expliquer cette tendance. Il est possible que, dans ces PME innovantes, l'innovation se diffuse à d'autres domaines. En cas de croissance forte, elles cherchent à retenir les salariés dont elles exigent plus tout en leur facilitant la vie. Ainsi le meilleur soutien à la conciliation travail-famille tiendrait à une culture d'entreprise d'innovation se préoccupant du bien-être général de chacun.

\section{La conciliation emploi-famille par les entrepreneures}

Pour les entrepreneures en TPE, dont la majorité vit maritalement (73\%) avec des enfants à charge $(52 \%), 38 \%$ des mères ayant créé leur entreprise après ou dès l'arrivée de l'enfant (Caisse d'Epargne, $2012: 13$ ), se posent la question de la conciliation vie privée-vie professionnelle. Ce thème a surtout fait l'objet d'études comparées femmes/hommes sous l'angle de la performance et de la croissance. Fitzgerald et Winter (2001) rappellent que la conciliation nécessite pour les entrepreneurs, hommes ou femmes, travaillant à domicile un véritable apprentissage de la gestion du temps, la vie familiale étant perturbée, entre autres, par les appels téléphoniques professionnels. L'autonomie dans l'organisation de leur temps est largement tempérée par un rythme soutenu et une durée de travail bien supérieure à celle de leurs homologues salariées (APCE, 2007). Le niveau d'activité des entrepreneures serait influencé par le temps disponible pour le travail, le soutien de la famille, la satisfaction au travail, le statut matrimonial et l'harmonie dans le couple (Nikina, Le Loarne et Shelton, 2012). Jennings et McDougald (2007) émettent l'hypothèse que la petite taille des entreprises dirigées par des femmes (en nombre de salariés et en bénéfices) serait le reflet d'une stratégie réfléchie de contrôle/limitation de la croissance par les entrepreneures qui vivent plus intensément le conflit travail-famille. A l'inverse, les études européennes insistent sur le fait que nombre d'entrepreneures font le choix de la croissance et de la conciliation travail-famille à travers plus de délégation, de partage ou de réduction de leur rôle dans l'entreprise que les hommes (Kirkwood et Tootell, 2008 ; Richomme-Huet, 2013 ; Léger-Jarniou, 2013).

Les femmes semblent plus sensibles aux éléments non monétaires du statut d'entrepreneur que les hommes selon Clain (2000) et, avant la création, elles étaient plus souvent employées et moins souvent cadres que les hommes (l'Entente des Générations pour l'Emploi et l'Entreprise ou EGEE, $2008: 10)$. Le récent courant de recherche sur les mampreneurs s'intéresse à la parentalité comme déclencheur de l'entreprenariat (D'Andria et Gabarret, 2014 ; Duberley et Carrigan, 2013; Richomme-Huet et D’Andria, 2013). La mampreneur est une femme qui crée son entreprise dans le même temps où elle devient mère ou qui est mère d'enfants pré-scolaires. Plus jeunes et souvent plus instruites que la moyenne des femmes entrepreneurs, la plupart ont quitté leur emploi salarié afin d'atteindre un équilibre vie professionnelle-vie privée qu'elles ne trouvaient pas dans le salariat. Leurs opportunités d'affaires sont souvent liées à la maternité (Harris, Morrison, Ho et Lewis, 2008). Si l'objectif premier de la majorité des entrepreneurs est d'assurer son propre emploi $(60 \%$ des hommes et $70 \%$ des femmes, selon l'EGEE, $2008: 9)$ et si la proportion de femmes «indépendantes sans 
salarié » est plus importante (66\%) que celle de leurs homologues masculins (57\%) (Banque Publique d'Investissement, 2014), cela n'est pas synonyme « d'entrepreneur par nécessité ». En effet, ces derniers sont définis stricto sensu par l'absence d'alternatives pour trouver un travail (Bosma et Levie, 2009), par exemple dans une situation de chômage, de décès ou de dettes dans la famille (Cornet, 2004). Les enquêtes s'accordent sur le fait que les entrepreneures par nécessité concernent surtout des situations d'isolement (femmes seules avec enfants ou immigrées) (Nakara et Fayolle, $2012: 235)$. La proportion de femmes entrepreneurs par nécessité est minoritaire et varie selon les enquêtes de $2 \%$ à $10 \%$ (Agence pour la Création d'entreprise ou APCE, 2007 :13) à 20\% (EGEE, 2008 :9) ou 25\% (Cornet, 2004 :197).

Une spécificité de l'entrepreneuriat féminin tient à l'importance du rôle du conjoint parmi l'ensemble des parties prenantes du projet (Nikina, Le Loarne et Shelton, 2012). Ainsi, la femme consulte son conjoint avant toute décision de nature entrepreneuriale (Brush, 1992). Dans l'échantillon de Kirkwood (2008) aucune femme ne créé son entreprise sans le soutien et l'accord de son conjoint - contre 15\% des hommes. En effet, le conjoint peut faciliter ou entraver l'entrepreneuriat (Daviddson et Honig, 2003).

Nikina, Le Loarne, et Shelton (2012) ont étudié les liens entre la relation de couple, le support du conjoint et l'entrepreneuriat féminin. Ils ont mis en évidence quatre configurations à l'intersection de deux axes: couple harmonieux/conflictuel et couple traditionnel/nontraditionnel, qui affectent à la fois le type et la nature du soutien du conjoint à l'entrepreneure et, en retour, comment l'entrepreneuriat féminin améliore ou détériore la relation de couple.

L'articulation travail-famille des femmes semble donc largement influencée par la représentation de leurs besoins, le type d'entreprise où elles exercent ainsi que le niveau de soutien de leur entourage. Pour notre étude, nous avons choisi le contexte français où les dispositions légales, relativement similaires entre cadres et dirigeantes et ancrées depuis 70 ans, permettent une interruption de leur activité.

\subsection{Les dispositifs légaux de congé en lien avec la maternité}

Le congé de maternité est un droit protecteur de la femme enceinte dans son emploi et de l'enfant à naître. C'est aussi un instrument censé faciliter l'articulation entre vie professionnelle et vie familiale dont il convient de définir les contours. Certains pays européens ont opté pour un système universel de protection sociale sans distinction entre les salariées et les indépendantes ; à l'inverse, la France se caractérise par un régime autonome de Sécurité sociale spécifique aux travailleurs indépendants.

Profondément ancré dans la culture française, le congé maternité est un droit relativement ancien pour les femmes salariées (Pretot, 2011). En effet, une loi de 1909 a posé les premières fondations d'un repos obligatoire non rémunéré de huit semaines à l'occasion de l'accouchement (Battagliola, 2009). Sur fond de politique familiale, des textes législatifs et réglementaires sont progressivement venus renforcer les droits accordés à la femme enceinte pour atteindre en 1970 une indemnisation par la Sécurité sociale du Régime général. Aujourd'hui, le congé maternité pour les femmes salariées se présente de la façon suivante : un congé appelé prénatal de six semaines avant la date présumée de l'accouchement et un congé postnatal de dix semaines après celui-ci (L 331-1 du Code de la Sécurité sociale). Ces mêmes congés peuvent se prolonger si l'assurée assume déjà la charge d'au moins deux enfants ou en cas de naissances multiples. L'assurée ayant cessé son activité est indemnisée par la Sécurité sociale (R331-5 du Code de la Sécurité sociale) pour un montant maximum de 82,33€ par jour en 2015 - souvent complété par l'employeur. Il faut noter que le congé maternité s'inscrit dans un dispositif particulièrement protecteur - en apparence à tout le moins - pour l'intéressée puisqu'il permet à la femme enceinte d'être protégée dès l'embauche, pendant sa période d'essai (L 1132-1 du Code du Travail) et l'exécution de son contrat de travail (R 241-50 du Code du 
Travail) jusqu'au retour à son poste (L 122-26 du Code du Travail). Par ailleurs, les femmes salariées ont la possibilité de bénéficier d'un congé parental d'éducation. Ce congé peut être pris jusqu'aux 3 ans de son enfant, l'employeur ne peut s'opposer à cette demande et, ceci quelle que soit la taille de l'entreprise (L 1225-47 du Code du Travail).

La femme chef d'entreprise est automatiquement inscrite au Régime Social Indépendant. Ses droits sont plus récents (loi du 12 juillet 1966 ; Chaudat, 2007). Deux types d'allocations, indépendantes l'une de l'autre, lui permettent de percevoir des revenus compensatoires pendant sa grossesse (L 613-19 du Code de la Sécurité sociale). Une première allocation, le repos maternel, est versée en deux temps, une première moitié à la fin du septième mois de grossesse et l'autre moitié après l'accouchement, son montant est fixé d'une façon forfaitaire à hauteur de $3129 €$ pour l'année 2014 (D 613-4-1 du Code de la Sécurité sociale). Cette allocation n'incite pas la femme chef d'entreprise à s'arrêter puisqu'elle n'est en aucun cas soumise à une obligation de condition d'arrêt de travail. A l'inverse, le versement de la deuxième allocation, appelée indemnité journalière forfaitaire, est conditionnée par la cessation de l'activité professionnelle pendant une période d'au moins quarante-quatre jours consécutifs dont quatorze jours doivent immédiatement précéder la date présumée de l'accouchement (D 613-4-2 du Code de la Sécurité sociale). Cette période d'indemnisation peut être prolongée à la simple demande de l'assurée pour une ou deux périodes de quinze jours consécutifs, Pour l'année 2014, le montant des indemnités est de 2262,92€ pour 44 jours d'arrêt, 3034,37€ pour 59 jours d'arrêt et $3805,82 €$ pour 74 jours d'arrêt. D'autre part, le régime de Sécurité sociale des Travailleurs Indépendants prévoit, lui aussi, une durée de versement des indemnités allongée de trente jours consécutifs en cas d'état pathologique résultant de la grossesse ou de l'accouchement attesté par un certificat médical en cas de naissances multiples (D 613-4-3 du Code de la Sécurité sociale).

Le droit au congé de maternité présente une durée et un taux d'indemnisation relativement similaires pour les entrepreneures et les salariées. Le congé parental n'existe pas chez les travailleurs indépendants mais il est relativement faiblement indemnisé pour les salariées et les durées d'obtention ont été durcies ${ }^{1}$. Cette concordance des droits permet ainsi une mise en perspective des discours de ces jeunes femmes selon une méthodologie qualitative comparative détaillée dans la partie suivante. Ces jeunes femmes à fortes responsabilités mais de statut différent (les unes dirigeantes, les autres cadres) partagent-elles les mêmes représentations de l'entreprise et de la maternité ?

\section{La méthodologie suivie}

Notre étude vise à explorer les représentations de femmes à fortes responsabilités face à la maternité, dans le contexte de PME (1 à 249 salariés), voire de TPE (1 à 9 salariés) en croissance. Nous avons privilégié deux secteurs d'activité en développement et prisés par les femmes (Belle, Novella, 2010) pour sélectionner le groupe de dirigeantes. Le secteur du commerce électronique (e-business) nous a semblé intéressant tant par sa capacité à innover que par les opportunités qu'il peut offrir en termes d'aménagement du temps de travail. L'autre secteur est celui des services à la personne, plus contraignant en termes de disponibilité (Smaili, 2002 ; Ratté, 1999). Les cadres en PME sont issues de cinq promotions d'une Ecole Supérieure de Commerce de province, soit 55 cadres en PME au moment de l'enquête sur 723 femmes diplômées. Compte-tenu des biais possibles, cela peut signaler un intérêt modéré de cette population pour les petites et moyennes entreprises. Neuf cadres exercent dans le secteur des services (conseil, immobilier, nouvelles technologies) et trois dans l'industrie (plasturgie, luxe

\footnotetext{
${ }^{1}$ La loi $\mathrm{n}^{\circ} 2014-873$ du 4 août 2014 oblige le père à prendre une partie du congé pour bénéficier d'une durée totale de congé indemnisé de 1 an ou de 3 ans respectivement pour le $1^{\text {er }}$ et le $2^{\text {ème }}$ enfant.
} 
et cosmétiques). Parmi les dirigeantes, huit exercent dans les services à la personne, deux dans les nouvelles technologies, une dans le commerce, une dans la communication - toutes exercent donc dans le secteur des services.Nous n'avons retenu que les femmes qui pouvaient prétendre à leurs droits en termes de maternité ${ }^{1}$ et dont la structure avait connu une hausse de $20 \%$ du chiffre d'affaires ou de l'effectif en trois ans, deux critères de croissance couramment retenus selon Janssen (2005). Il s'agit ici de données déclaratives. Entre mars et mai 2013, nous avons interrogé par téléphone vingt-quatre femmes sur toute la France, nées entre 1978 et 1988, soit âgées de 25 à 35 ans au moment de l'enquête. En effet, l'âge moyen des Françaises à l'arrivée du premier enfant est de 28,1 ans ; le deuxième enfant naît approximativement 3,9 ans après le premier (Davie, 2012).

Les caractéristiques des femmes interrogées présentent les similarités suivantes : une moyenne d'âge de 32 ans, diplômées d'études supérieures (exceptée une dirigeante), en couple, avec un enfant à charge pour la majorité d'entre elles, et avec d'importantes responsabilités depuis au moins deux ans dans l'entreprise. Les deux groupes se différencient par le statut de l'interviewée (dirigeante ou cadre salariée), la taille moyenne des entreprises (TPE pour la majorité des dirigeantes, contre 4 TPE et 4 entreprises de 100 à 250 salariés pour les cadres), et des secteurs d'activité, plus masculins pour les cadres (conseil, industrie), plus féminins pour les dirigeantes comme les services à la personne.

Tableau 1. Variables descriptives des échantillons :

\begin{tabular}{|c|c|c|}
\hline & 12 femmes dirigeantes & 12 femmes cadres \\
\hline Age & Entre 25 et 35 ans, moyenne : 33 ans & $\begin{array}{l}\text { Entre } 26 \text { et } 35 \text { ans, moyenne d'âge de } 31 \\
\text { ans. }\end{array}$ \\
\hline $\begin{array}{l}\text { Situation } \\
\text { familiale }\end{array}$ & En couple, avec au moins un enfant & $\begin{array}{l}\text { En couple, la moitié avec au moins un } \\
\text { enfant }\end{array}$ \\
\hline $\begin{array}{l}\text { Revenus du } \\
\text { couple }\end{array}$ & Autour de $2500 €$ net par mois & Entre $2500 €$ et $3500 €$ net par mois \\
\hline Diplômes & $\begin{array}{l}11 \text { diplômées d'au moins un niveau L3. } \\
\text { Une seule femme n'a pas le } \\
\text { baccalauréat }\end{array}$ & $\begin{array}{l}\text { Diplômées bac }+5 \text { d'une Ecole de } \\
\text { commerce de province }\end{array}$ \\
\hline $\begin{array}{l}\text { Situation } \\
\text { professionnelle } \\
\text { du conjoint } \\
\end{array}$ & Essentiellement des cadres & 8 cadres, 4 non-cadres \\
\hline $\begin{array}{l}\text { Localisation de } \\
\text { l'entreprise }\end{array}$ & Villes de province, 5 en Ile de France & Villes de province, 2 en Ile de France \\
\hline $\begin{array}{l}\text { Croissance de } \\
\text { l'entreprise }\end{array}$ & $\begin{array}{l}\text { Déclaratif : CA en hausse régulière } \\
\text { chaque année, } 20 \% \text { sur } 3 \text { ans. Pour une } \\
\text { PME, le CA a doublé en } 6 \text { mois }\end{array}$ & $\begin{array}{l}\text { Hausse du CA de } 20 \% \text { et de l'effectif ( } 10 \\
\text { cas), sans modification de l'effectif dans } 2 \\
\text { cas. }\end{array}$ \\
\hline $\begin{array}{l}\text { Taille de } \\
\text { l'entreprise }\end{array}$ & 11 TPE et 1 entreprise de 250 salariés & $\begin{array}{l}4 \text { TPE, } 3 \text { entreprises de } 30 \text { à } 50 \text { salariés, } 4 \\
\text { entreprises de } 100 \text { à } 250 \text { salariés }\end{array}$ \\
\hline $\begin{array}{l}\text { Secteur } \\
\text { d'activité }\end{array}$ & E-commerce. Services à la personne & Conseil / Industrie \\
\hline
\end{tabular}

\footnotetext{
${ }^{1}$ A la date de la naissance de l'enfant, la salariée doit justifier de dix mois d'immatriculation et avoir effectué au moins 200 heures de travail salarié au cours des trois mois civils précédant l'accouchement ( $\mathrm{R}$ $313-3$ du Code de la Sécurité sociale) ou d'un an d'ancienneté pour bénéficier du congé parental (L 1225-47 du Code du Travail). Aucune ancienneté d'immatriculation n'est demandée pour la dirigeante.
} 
Des entretiens semi-directifs intégralement enregistrés de quarante-cinq minutes à une heure, ont été menés par téléphone à l'aide d'un guide d'entretien. Celui-ci regroupe trois thèmes. Un premier thème relatif à des «variables descriptives », telles que la fonction exercée pour les cadres en PME, l'âge, le niveau de diplôme, le niveau des revenus du couple, la situation de famille, la situation professionnelle du conjoint, le descriptif de l'entreprise (secteur, localisation, etc.). Un deuxième thème se rapporte à la connaissance des droits en matière sociale et à la prise du congé maternité/parental. Enfin, un troisième thème s'intéresse aux motivations pour prendre le congé ou non, le rôle de l'entourage, les perspectives et les aspirations de carrière.

Après transcription, nous avons réalisé une analyse thématique de contenu sur ces entretiens répandue pour ce type de données qualitatives (Point et Retour, 2009; Thiétart, 2006). Nous avons également eu recours à des données secondaires, telles que de la documentation interne, les pages web des structures concernées et les données RSI nationales et Auvergne.

\section{Principaux résultats}

Notre étude porte sur les représentations de la maternité et de l'entreprise de jeunes femmes à fortes responsabilités (dirigeantes et cadres) dans des entreprises petites et moyennes en croissance. Leurs représentations suivent-elles plutôt une perspective négative, positive ou de co-évolution? Partagent-elles les mêmes représentations ? Leur connaissance de leurs droits estelle la même ? L'analyse de nos entretiens montre que ces représentations se déploient autour de cinq thématiques : une représentation différenciée de l'entreprise, qui ne représente pas le même type d'opportunité de concilier maternité et vie professionnelle, la perception du rôle de l'entourage, la maternité perçue comme frein/levier/mise en veilleuse des objectifs professionnels, enfin la connaissance et l'usage des droits. En raison d'une grande richesse des transcriptions, seuls les verbatim les plus significatifs sont reproduits.

\subsection{Une représentation différenciée de l'entreprise selon les postures professionnelles}

\section{La création d'entreprise : une procréation?}

Invitées à présenter la structure dans laquelle elles exercent, les entrepreneures et les cadres mettent en lumière leur univers professionnel de façon subjective: elles évoquent spontanément comme caractéristiques l'autonomie et la flexibilité. Alors que les cadres citent la diversité des tâches au travail, les entrepreneures insistent sur l'articulation travail-hors travail. La recherche d'autonomie et de flexibilité font partie des principales motivations de la création d'entreprise (Mattis, 2000 ; Lambrecht, Pirnay, Amedodji et Aouni, 2003 ; Carrier, 2006). Pour ces mampreneurs qui s'identifient à la fois comme mère et femme d'affaires (Richomme-Huet et D'Andria, 2013), cela implique une sorte d'osmose des lieux et des temps professionnels et familiaux :

Marion indique avoir «aménagé mon bureau, pour que mon petit garçon puisse venir faire ses devoirs pendant que maman travaille». Chloé rappelle que «Lilou a fait ses premiers pas dans l'entreprise ».

Il est frappant de constater que, pour la majorité des mampreneurs, que la création d'entreprise est relatée dans l'entretien comme une forme de maternité et qu'elles en ont parlé comme de l'un de leurs enfants. Avec cette analogie, l'entreprise est ainsi attendue, choyée, 
accompagnée par son entourage jusqu'à son terme, parfois d'une manière égale à l'enfant attendu.

«L'entreprise est mon bébé, je l'ai attendue et construite. » (Audrey)

«J'étais enceinte quand j'ai créé mon entreprise et mes deux jumelles [l'une est son entreprise] sont nées à 3 mois d'intervalle, ma petite fille d'abord puis mon entreprise. »(Chloé)

«Le fait d'avoir eu un enfant, a été une prise de conscience, j'ai compris que la boîte a été mon premier bébé, mon enfant a été mon deuxième bébé. » (Aurélie).

C'est ce que relève également Bah (2009) concernant les cédants d'entreprise qui peuvent exprimer « un refus conscient, ou non conscient, de perdre un objet d'amour central dans la construction de leur identité personnelle et sociale ». L'entreprise est perçue comme une prolongation d'eux-mêmes (Pailot, 2002).

On pourra s'étonner de la présence forte de ce thème dans ces entretiens. L'étude abordant la question de la maternité a sûrement facilité l'utilisation d'une terminologie croisée mais l'analogie est si forte en termes de symbole et de régularité dans les discours qu'il est à souligner et à interroger : est-ce une façon d'argumenter contre un sens commun qui voudrait que la femme donne toujours la priorité à la maternité? Si l'on préfère, de telles affirmations peuvent s'entendre comme une sorte d'autojustification de leur choix d'investissement professionnel. Ou est-ce le pendant féminin de la «création»? En tout cas, dans cette configuration, les données que nous avons recueillies mesurent toute l'importance de l'attachement émotionnel. Les femmes cadres quant à elles n'emploient jamais cette analogie. La personnification de l'entreprise considérée comme un enfant a d'importantes répercussions sur la légitimation d'un mélange des temps et des lieux professionnels/familiaux pour les mampreneurs..

\section{Femmes cadres en PME : un choix assumé}

Certaines femmes cadres interrogées présentent, leur choix de l'entrée dans une PME comme motivé par l'exercice de responsabilités étendues avec une certaine autonomie, par la polyvalence des tâches. Ce choix permet en outre de conserver la proximité géographique avec la famille étendue, ce qui constitue un atout lorsque les enfants sont en bas-âge.

«J'ai refusé un poste dont le contenu ne me correspondait pas [dans une grande entreprise où s'est déroulé mon apprentissage] ... En PME, rien n'est figé, il y a la possibilité de créer des choses, de mettre en place des choses...La société est passée de 45 à 132 salariés en 5 ans. Il a fallu s'organiser, créer un certain nombre de process, monter des équipes. On est très libre de moduler notre poste... même si on a des objectifs très précis sur certaines choses [en matière de recrutement], à côté de cela, on a une grosse marche de manœuvre... ( (Cindy).

«Je savais exactement que je voulais travailler dans une agence de communication spécialisée sur le secteur financier [...] Je voulais une petite structure pour l'autonomie, la flexibilité, on apprend plus vite, on prend plus de responsabilités plus vite » (Marie).

«A la fin du premier entretien [dans un cabinet lyonnais de 150 salariés alors qu'elle attendait les réponses de grands cabinets], il m'a fait une proposition d'embauche et j'ai accepté tout de suite. En Big [sous-entendu grands cabinets], je n'aurais pas pu [faire $75 \%$ d'audit et $25 \%$ d'expertise], il aurait fallu que je me spécialise. Et dans les Big, il y avait toujours la possibilité qu'on m'envoie six mois, je ne sais pas, à Montpellier... J'ai choisi la proximité » (Carine). 
Les mampreneurs interrogées fournissent des exemples où l'autonomie et la flexibilité, en tant qu'entrepreneure, leur permettent de combiner les sphères professionnelles et privées. Pour les femmes cadres interrogées, l'autonomie et la flexibilité dans les tâches au travail participent de l'attractivité de la PME. Nous verrons dans la partie suivante que la perception des dirigeantes de l'opportunité de concilier la maternité et la vie professionnelle est globalement positive. Pour les femmes cadres interrogées, cette perception est plus contrastée.

\subsection{L'entreprise est l'occasion de concilier maternité et vie professionnelle notamment pour l'entrepreneure}

Nous avons choisi, dans nos entretiens, un focus sur la période qui va de la grossesse aux trois ans de l'enfant. Cette période de repos nécessaire et d'investissement auprès de l'enfant, oblige la mère à adopter des stratégies de conciliation entre maternité et vie professionnelle.

\section{L'opportunité d'un nouveau lien travail-famille aux yeux de l'entrepreneure?}

Pour les entrepreneures interrogées, ayant un projet de maternité ou lorsque l'enfant est né, le fait de gérer leur entreprise est présenté comme une véritable opportunité de concilier leur vie familiale et professionnelle. La croissance de l'entreprise n'est pas considérée comme une entrave à cette conciliation comme l'attestent d'autres études (Shelton, 2006; Jarniou-Léger, 2013). Plusieurs entrepreneures évoquent les avantages d'être à leur compte. L'absence de supérieurs hiérarchiques, la possibilité d'amener ses enfants sur son lieu de travail, l'occasion d'aménager son temps de travail, ou encore la possibilité de travailler de chez soi, à n'importe quelle heure y compris le soir très tard lorsque les enfants sont couchés, représentent des avantages précieux. Selon la théorie de la préférence des styles de vie ${ }^{1}$ (Hakim, 2000), la majorité des dirigeantes interrogées se positionnent dans le mampreneuriat afin de combiner travail et vie de famille (Richomme-Huet et D'Andria, 2013), Comme indiqué précédemment, la recherche d'autonomie et de flexibilité est ainsi l'une des principales motivations de la création d'entreprise. Cela confirme les travaux antérieurs (Mattis, 2000 ; Lambrecht, 2003 ; Carrier, 2006).

A titre d'exemple, pour Marion, l'entreprise a été une véritable «chance ». «J'ai pu m’organiser différemment. Mon métier me le permet, je vais chercher mon fils tous les jours à $16 \mathrm{~h} 30$ et je m'en occupe tout le mercredi. Je travaille plus le soir et plus le weekend. J'ai aménagé mon bureau, pour que mon petit garçon puisse venir faire ses devoirs pendant que maman travaille ». Une autre femme, Chloé rappelle que «Lilou a fait ses premiers pas dans l'entreprise ». Charlotte indique qu' «avec les portables et internet, cela aide beaucoup, on peut travailler de chez soi, c'est une question d'organisation »; ou enfin une autre femme, Jeanne précise que « rien n'est impossible avec des enfants, il suffit de vouloir ».

Le projet de maternité des femmes entrepreneurs s'inscrit dans une stratégie globale de conciliation, ce qui rejoint les travaux de Cornet (2004) et de Richomme-Huet (2013) sur les

\footnotetext{
${ }^{1}$ Trois types de style de vie se distinguent : (1) Celui où les femmes sont centrées sur leur famille et sur leurs enfants, la priorité est donnée au foyer. (2) Celui des femmes adaptatives qui cherchent à combiner le travail et la vie de famille, en se positionnant de préférence sur des professions qui facilitent cet équilibre. (3) Celui des femmes qui donnent la priorité à leur le-travail ou à une activité équivalente en terme de carrière.
} 
mampreneurs. La maternité se présente ici comme l'élément déclencheur de la création de l'entreprise. C'est le cas de Leila, soumise à de nombreux déplacements dans le cadre de son activité de salariée, elle réalise que la crèche ne peut répondre à ses exigences : trop peu de places, demande croissante, système de gardes rigide, prestations peu développées. La maman a alors créé une entreprise aujourd'hui forte de 250 salariés.

«A la base, je me suis retrouvée maman, j'étais enceinte d'une petite fille et vu le métier que j'exerçais avant, il y avait beaucoup de déplacements, je n'allais pas pouvoir faire garder ma fille sereinement. Comme je ne trouvais pas de mode de garde pour ma fille, je me suis dit que j'allais créer ma boite dans ce secteur; j'avais des exigences, le système d'assistante maternelle ne me convenait pas du tout, surtout que Montpellier est une ville en travaux depuis vingt ans. Aller déposer son enfant le matin et faire le tour de la ville était impossible. J'ai donc créé ma boîte dans le secteur des gardes d'enfants » (Leila).

Notre recherche relate que l'entreprise a été une opportunité de concilier les affaires et la maternité confirmant ainsi ce que soulignent les travaux de Harris, Morrison, Ho et Lewis (2008).

\section{La PME en croissance perçue par les cadres comme une opportunité peu conciliable avec l'arrivée d'un enfant.}

A l'inverse, la perception des jeunes femmes cadres apparait nettement plus contrastée. Entrées depuis cinq à dix ans sur le marché du travail, ces jeunes femmes diplômées bac+5 continuent d'entretenir de fortes aspirations pour «toujours progresser dans le métier, développer de nouvelles compétences, apprendre de nouvelles choses ». Certaines envisagent à terme de monter leur propre structure, de s'associer ou de reprendre l'entreprise familiale d'un ascendant (Marie, Lucie, Rose, Léa, Zoé, Cindy). A deux exceptions près, toutes aspirent à prendre plus de responsabilités, et à changer de structure si nécessaire. Ces cadres ont cherché de fortes responsabilités en PME et se trouvent «prises au jeu» (ou prises au piège) de la croissance de la structure. Dans 10 PME sur 12, la hausse des effectifs est intervenue simultanément à celle du chiffre d'affaires. La croissance de l'effectif a ouvert des possibilités d'évolution de carrière dans la PME, présentée comme difficilement compatible avec l'arrivée d'un enfant. Cet élément était absent des discours des deux cadres exerçant en PME où il n'y a pas eu de changement d'effectif important. Toutes expriment le souhait de continuer à travailler à temps plein « dès leur retour » ou « dès que possible » après une naissance. Il y a là une forte intériorisation des attentes de l'entreprise.

« Je ne voudrais pas trop louper quelque chose en m'absentant trop longtemps...et je sais que moi, je ne voudrais pas non plus 'louper le coche', enfin louper quelque chose au niveau de la carrière. » (Léa)

Deux cadres indiquent que leurs fonctions RRH dans une PME où les effectifs sont en croissance réclament leur présence. A leurs yeux, même un temps partiel à $90 \%$ ne serait pas compatible avec leur fonction et leur charge de travail en période de croissance d'effectif. Le télétravail n'est donc pas une option pour elles. En outre, elles invoquent l'absence de compétences en interne pour assurer leur remplacement.

Les femmes cadres de notre échantillon, qui exercent majoritairement dans des environnements masculins, partagent ici avec les hommes cadres une vision en faveur d'une poursuite du travail à temps plein après l'arrivée de l'enfant. Nous tenons à souligner que cela ne nous semble pas lié à des facteurs individuels (contraintes matérielles ou organisationnelles), 
mais plutôt à une même attitude pour appréhender le possible et le désirable. Il nous semble approprié d'appliquer ici le concept « d'ethos » quand elles évoquent le congé parental - au sens de dispositions acquises qui orientent les pratiques et les jugements de valeur sur ces pratiques, suite à une intériorisation, comme sous l'effet du regard des autres (Fuselier, 2011b ; Zarca, $2009: 352$ )

«Pour moi, c'est compliqué [un congé parental] : les gens sont très attachés à la présence du responsable RH. » (Pascale) En présentant le cas d'une autre cadre qui en a bénéficié dont elle parle comme un contre-exemple de ses propres choix : "Cela a demandé une réorganisation quand même. » (Pascale)

«Pour vous-même et pour l'entreprise, ce n'est pas toujours simple; je pense qu'il ne faut pas non plus «perdre la main » au niveau du travail. » (Léa)

«C'est quelque chose qui m'aurait tentée mais qui me fait peur par rapport au maintien de ma position. » (Anne)

Il paraît opportun de faire observer ici que l'entretien est une sorte de scène où se joue une représentation de soi dont l'enjeu est la valorisation de celle qui parle (et l'importance de sa position dans l'entreprise) face au tiers que constitue l'enquêtrice. Lorsque Pascale affirme que les autres ont besoin d'elle, c'est, en somme, son devoir moral à l'égard des autres et de l'entreprise qu'elle rehausse. Rien n'autorise à en conclure qu'elle tiendrait toujours et partout le même discours, avec les mêmes attendus. Si l'on préfère, les prises de position traduisent ici un espace symbolique de légitimité structuré autour du statut : la prise de congé parental légal a une faible légitimité parmi les cadres.

Les femmes cadres qui ont des fonctions commerciales pressentent que les objectifs sont non-négociables.

« Ici, tous les acheteurs sont passés cadres en même temps, en raison des missions et aussi des horaires surtout, la direction veut ainsi éviter de payer des heures supplémentaires. Je ne sais pas si la charge de travail est compatible avec un congé parental, donc je préfère ne pas en demander. Je crains que les objectifs ne soient pas revus à la baisse.... » (Sarah)

Une cadre indique avoir été licenciée juste après la période légale de son retour de congé maternité. Elle n'ose pas aborder le sujet avec son nouvel employeur avec la venue du $2^{\text {ème }}$ enfant bien qu'elle soit tentée par un congé parental à $80 \%$.

«La première expérience que j'ai eue m'a marquée. J'ai débuté comme responsable export, seule femme salariée d'une PME industrielle de 80 personnes. J'ai eu mon enfant et trois mois après, j'étais licenciée. [un congé parental à $80 \%$ ] m'aurait tentée mais [cela] me fait peur par rapport au maintien de ma position... sans parler d'évolution même, mais de mon maintien dans l'entreprise.» (Anne)

A cet effet, nous rappelons que le baromètre IFOP de janvier 2014 sur la perception de discrimination au travail indique que la maternité et la grossesse interviennent dans $20 \%$ des cas de discriminations au travail. Les rapports officiels font état d'un nombre toujours croissant de plaintes de salariées pour discriminations liées à la grossesse (La Halde, 2011).

En bref, il ressort de nos entretiens que certaines cadres disent que leur emploi pourrait être menacé. Leur emploi de cadre, tant prisé pour l'autonomie dans les tâches, ne leur permet pas d'être réellement maîtres de leur temps de présence, de leurs objectifs et, au final, de leur organisation du temps entre sphères privée et professionnelle. Majoritairement, ces cadres ont choisi de retarder l'arrivée du premier enfant afin d'avancer dans leur carrière. 
« Je n'ai pas souhaité avoir d'enfant pour l'instant par rapport à ma carrière.» (Pascale).

«Pour vous-même et pour l'entreprise, ce n'est pas toujours simple ; je pense qu'il ne faut pas non plus «perdre la main » au niveau du travail » (Léa).

«Cela tient à notre métier plus qu'au statut, donc on a une mission, il faut la mener à bien et mettre le temps qu'il faudra... [l'arrivée d'un enfant], vu mon métier, ça va être compliqué, pour l'instant c'est comme cela que je le vois » (Marie).

Deux cadres de notre échantillon ont toutefois pris un congé parental pour mieux articuler vie de famille et vie professionnelle. Carine a pris un congé parental d'un an à temps plein qui a été l'occasion d'engager une reconversion réussie vers le commercial. Rose s'est d'abord assurée de l'accord de la dirigeante pour un temps partiel aménagé et s'est toujours montrée flexible et disponible lorsque l'activité le nécessitait :

«Après on s'adapte. Je travaillais de chez moi quand même, je n'étais pas complètement coupée de tout ce qui se passait... et puis parce que je ne voulais pas laisser tomber complétement l'activité et qu'il y ait des soucis ensuite »(Rose).

Ainsi la perception de l'entreprise comme opportunité (ou non) d'articuler la maternité et la vie professionnelle semble relativement clivée autour du statut de l'entrepreneur ou de celui de salarié. Nous allons voir que la réussite de cette articulation s'avère également tributaire de l'investissement de l'entourage proche de l'intéressée.

\subsection{L'entourage proche, levier essentiel d'une réussite professionnelle ?}

\section{L'entourage, un facteur de succès en entrepreneuriat pour les entrepreneures}

Les entrepreneures ont associé à plusieurs reprises leur entourage à la réussite de la conciliation de leur vie familiale avec leur vie professionnelle. C'est encore plus prégnant dans un contexte de TPE en croissance qui génère un investissement particulièrement important de l'entrepreneure. Majoritairement, l'entourage se résume au conjoint. Certaines femmes ont également évoqué un soutien plus large, comme les parents, les frères, les sœurs, un réseau d'amis.

«L'entourage est la clef numéro un» (Chloé).

Chloé ne peut se consacrer seule à ses différentes tâches familiales. Cela corrobore les travaux relatés dans la littérature à ce sujet. L'articulation travail-famille semble facilitée pour les entrepreneures dont le conjoint présente une vision moderne du rôle de la femme dans la famille et dans la société et où le couple vit en harmonie (Carrier, 2006, Boutillier, 2008 ; Nikina, Le Loarnes et Shelton, 2012).

Dans notre échantillon, le conjoint est présenté comme important par son soutien à la fois émotionnel, moral (maintien de l'estime de soi) et matériel (les tâches ménagères, l'aide apportée aux autres membres de la famille) et ses encouragements dans la carrière. La nature de ces soutiens renvoie à la typologie d'Ezzedeen et Ritchey (2008).

«J'ai été portée par mon mari, ma famille, j'ai toujours eu de la bienveillance » (Elodie).

« J'ai eu la chance d'avoir un mari formidable et extraordinaire, avec lequel on a porté le projet, qui a toujours été là dans les hauts et dans les bas» (Chloé). 
«On a une règle à la maison, c'est chacun à son tour, le lundi, j'étais à Lille, aussi mon conjoint a pris le relais, mes deux enfants le savent. » (Jessica).

D'ailleurs, parfois, le conjoint mesure encore mieux la charge de travail et joue le rôle de support dans la carrière du conjoint puisqu'il est lui-même entrepreneur et père de famille.

«L'entreprise m'a permis d'adapter mes horaires, je ne travaille pas un mercredi et mon compagnon qui est également travailleur indépendant assure l'autre mercredi » (Audrey).

«Quand les enfants sont malades et que je suis en réunion, c'est lui qui s'y colle ...on a un vrai partage du temps familial et c'est une vraie chance » (Pauline).

En bref, ici le conjoint occupe une place active dans l'aide apportée aux membres de la famille. Il répond présent pour la garde du ou des enfants, pour la participation aux tâches ménagères, ou pour les tâches administratives (inscription à la crèche) ou médicales (visite médicale, soins médicaux ou pharmaceutiques).

\section{L'entourage, un soutien qui reste à confirmer pour les femmes cadres}

Les femmes cadres de notre échantillon vivent majoritairement en couple avec un cadre (10 cas sur 12, ce qui est cohérent avec les travaux de Vanderschelden (2006) sur l'homogamie parmi les femmes cadres). Elles sont dans une logique de cumul travail-famille, afin de préserver leur vie professionnelle et leur carrière. En premier lieu, les femmes de l'échantillon indiquent que leur conjoint leur a "laissé le choix »[ de suspendre ou d'interrompre leur carrière] à l'arrivée du premier enfant. C'était à elles de décider ce qu'elles voulaient faire. En réalité, le choix de ces femmes tient compte du fait que l'investissement professionnel du conjoint est une donnée fixe non négociable.

«L'idée d'un congé parental ne l'a même pas effleuré » (Cindy).

A nouveau, nous désirons souligner l'ethos professionnel des cadres, selon lequel il n'est pas légitime de travailler moins, qui s'observe cette fois à travers l'attitude contrastée des deux conjoints non-cadres, même s'il convient néanmoins de ne pas généraliser compte tenu de la taille réduite de notre échantillon. Les femmes avec des conjoints non-cadres ont tenu à nous donner la position de leur conjoint ayant exprimé le souhait de prendre un congé parental à temps partiel :

«C'est l'idée qu'il envisage de lui-même. [Et il s'arrêterait complètement ?] On n'a pas poussé aussi loin la réflexion. En tout cas, il n'est pas opposé au congé parental pour les hommes. C'est même quelque chose qui l'intéresse » (Lucie).

«Mon souhait est de ne pas m'arrêter... et mon mari ? Ah oui, vraiment, il voudrait bien, cela ne le dérangerait pas. Une fois, il m'a dit : «Au global, il vaut mieux que tu ailles travailler plutôt que moi » Donc je pense qu'il prendra le congé parental » (Léa).

Ces résultats corroborent ceux de Boyer (2004) qui montrait que les $2 \%$ de pères bénéficiaires de l'Allocation parentale d'éducation vivaient avec une conjointe dont le statut socioprofessionnel, le revenu professionnel et le diplôme étaient le plus souvent plus élevés que le leur. Boyer (2004) s'est d'ailleurs demandé si cette 'paternité active' n'était pas contrainte par les horaires de travail de la mère (horaires atypiques, temps complet).

Pour conclure, aucune des femmes entrepreneures et cadres interrogées n'a mentionné que leur conjoint chercherait à entraver leur carrière, ce qui rejoint les travaux de Daviddson et Honig (2003). Les entrepreneures ont de plus souligné les différentes formes de soutien par leur 
conjoint. Quant aux quatre femmes cadres mariées avec des non-cadres, elles ont même assuré que leur conjoint souhaitait prendre un congé paternité de longue durée. Quant aux six femmes cadres mariées avec un cadre, il ne leur suffit pas, d'après elles, d'avoir le même statut cadre et d'être également attachées au fait de travailler, pour voir leur conjoint adopter un comportement de «paternité active ». De même, ces femmes cadres «s'autorisent» rarement à prendre un congé parental.

\subsection{Une maternité revendiquée par les entrepreneures, considérée comme un frein par les cadres?}

\section{La maternité, un levier professionnel aux yeux des entrepreneures}

Les dirigeantes de TPE interrogées déclarent réussir à articuler maternité et leurs objectifs professionnels. C'est ainsi qu'elles présentent leur vécu, vraisemblablement aussi parce que la maternité n'a pas été subie mais choisie en collaboration étroite avec un entourage, lequel a été présent et actif dans la vie familiale de l'ensemble de notre échantillon.

«Il ne faut pas passer à côté des choses essentielles. Je pense que dans une vie de femme, si on n'est pas capable de donner trois mois à un enfant, même si on est chef d'entreprise, c'est qu'on passe quand même à côté de quelque chose » (Aurélie).

L'entrepreneure affirme ici que l'entreprise n'est pas un frein à son investissement maternel.

On voit à quel point la conception de «l'essentiel» (ou du devoir) peut se déplacer fortement du discours de la femme cadre citée supra, à celui de cette dirigeante. Les points de vue semblent opposés, tout en répondant, dans les deux cas, à une logique de la plus grande valorisation de soi sur la scène de l'entretien. Une autre femme entrepreneur tient un discours plus problématisé sur l'articulation maternité et objectifs professionnels.

«Mes enfants n'ont jamais été des freins. Sur un plan personnel, on réfléchit à un quatrième enfant, mais en même temps, j'ai vraiment envie de m'occuper de l'entreprise » (Sonia).

La maternité est donnée alors comme un projet personnel et conjugal vécu en parallèle au projet de développement de l'entreprise («on réfléchit») même si ces parallèles peuvent être contradictoires : un quatrième enfant, mais en même temps, l'entreprise. On ne sait pas ici si l'envie de s'occuper de l'entreprise risque d'être frustrée par l'arrivée du quatrième enfant qui ne lui permettra plus de s'investir comme auparavant - ou si l'envie de s'occuper de l'entreprise est liée à volonté de s'occuper plus de l'entreprise dans le futur qu'elle n'a pu le faire dans le passé - donc une frustration antérieure qu'elle cherche à dépasser.

Dans tous les cas, l'entrepreneure explique qu'elle s'est organisée à sa manière. Ainsi Jeanne témoigne de ce moment particulier de son accouchement : «Quand mes enfants sont arrivés, j'ai continué à travailler autant, j'ai dû m'organiser autrement. Une heure avant d'accoucher, j'étais en réunion clientèle et à la sortie de la maternité, j'avais mon PC pour pouvoir répondre aux clients qui attendaient des réponses ». En choisissant de parler ainsi de ce point de son histoire, cette interviewée met en valeur sa capacité à faire face et illumine un vécu quotidien qui n'a sans doute pas été aussi facile avant ou après. Elle se dispense ainsi de préciser en quoi consiste ce « devoir s'organiser autrement », insistant sur la possibilité du télétravail.

\section{La maternité perçue comme une entrave à la carrière des cadres en}




\section{PME en croissance?}

Une seule femme cadre, sur les douze interrogées, a présenté son congé (maternité et parental) comme l'occasion d'une reconversion personnelle : elle a démissionné de son poste d'expert-comptable/audit pour se reconvertir et postuler sur un poste de chargée d'affaires dans une entreprise de logiciels spécialisés sur ce marché. Les cadres, du fait de leur position salariée, ont exprimé pour la plupart l'inquiétude des conséquences d'une maternité sur leur carrière, de façon plus ou moins explicite.

«Personne n'est irremplaçable » (Lise)

«Cela me fait peur par rapport à mon maintien dans l'entreprise » (Anne).

« Je ne veux pas perdre la main au niveau du travail » (Lea).

« Enfin, je n'ai pas fait toutes ces études pour gâcher ça. » (Zoé).

Ces verbatim expriment la peur de perdre son emploi ou son poste avec la crainte d'un possible déclassement (Lise, Anne, Zoe), l'appréhension de l'éloignement lié à une perte d'agilité ou de compétences dans un contexte en évolution, enfin la crainte de voir leur échapper des opportunités de carrière émergeant du seul fait de la croissance de la PME. Ainsi, à l'inverse des entrepreneures, la plupart des femmes cadres interrogées indiquent que prendre un temps partiel ou s'arrêter de travailler lors de la naissance d'un enfant dans une PME en croissance constitue à leurs yeux un frein à leur carrière. Que cette opinion repose ou non sur une réalité, il n'en demeure pas moins que ces craintes exprimées et les représentations qui les nourrissent s'imposent à elles.

Le fait qu'une maternité soit revendiquée par les dirigeantes et considérée comme un frein par les cadres peut expliquer une différence de connaissance et de l'usage des droits liés à la maternité. Ce sera l'objet de notre dernière partie.

\subsection{Une connaissance des droits confirmée chez les entrepreneures, et réinterprétée par l'ethos des cadres.}

\section{La connaissance et l'usage des droits chez les entrepreneures}

Les entrepreneures connaissent leur régime de Sécurité sociale et leurs droits dans leur ensemble. Les 12 femmes interrogées les ont toutes utilisés. A noter qu'au niveau national $90 \%$ des dirigeantes interrompent leur activité professionnelle avant ou après l'accouchement et utilisent leurs droits (RSI, 2012). Il ne nous est pas apparu de difficultés de compréhension à l'égard de l'allocation de repos maternel ou des indemnités journalières d'interruption d'activité. Cela peut s'expliquer par une forte communication sur le sujet impulsée par le régime de Sécurité sociale des Travailleurs indépendants du fait d'une réorganisation des institutions (Chaudat, 2007).

« Je me suis renseignée sur ses droits, le Régime social des Indépendants communique beaucoup sur le congé de maternité» (Cécile).

« Je connaissais mes droits en la matière. Des campagnes d'informations ont eu lieu et j'ai utilisé l'ensemble des aides proposées » (Charlotte).

A titre de comparaison, en 2012 (RSI-Auvergne, 2013), sur les 228 grossesses de femmes chefs d'entreprise de la région Auvergne, 186 (81.5\%) ont bénéficié de l'allocation 
forfaitaire et $175(77 \%)$ ont perçu des indemnités journalières ${ }^{1}$. Pour ces mêmes femmes, le nombre moyen de jours indemnisés est de 79,3 jours, ce qui correspond à une période d'indemnisation similaire à celle du droit des salariées.

Par contre, un point important est à souligner : le bénéfice des allocations et indemnités n'engendre pas une déconnexion totale avec l'activité professionnelle. La réalité du terrain ne va pas dans le sens de la législation qui oblige la femme chef d'entreprise à interrompre son activité professionnelle pendant une période d'au moins quarante-quatre jours consécutifs dont quatorze doivent immédiatement précéder la date présumée de l'accouchement. Même si, physiquement, l'entrepreneure n'est pas présente dans l'entreprise, mentalement l'entrepreneure n'est jamais très loin de son « deuxième bébé ».

«Mon activité n'a pas cessé pendant mon arrêt de travail» (Jeanne). «J'ai eu la chance d'avoir une grossesse facile, ça m'a pas empêché de travailler pour mon entreprise. Mon entreprise est chez moi » (Aurélie).

Pour les entrepreneures, notre recherche met clairement en exergue que la frontière entre vie familiale et vie professionnelle est inexistante pendant la maternité particulièrement dans les entreprises dont le siège social est à la maison. Le contexte d'une TPE en croissance nous est apparu comme un élément amplificateur de cet investissement.

\section{Une connaissance floue et limitée chez les cadres}

A l'opposé, on note qu'un certain flou émerge des discours des femmes cadres concernant les dispositifs d'accès et d'indemnisation des congés liés à la maternité. Ces cadres ne se posent pas de questions.

« Je ne me suis pas posée la question, même quand j'ai eu ma fille » (Sarah).

Parfois amenées à côtoyer des salariées qui ont pris un congé parental, elles indiquent avoir reçu des informations « par hasard » (Rose, Carine).

Selon Fusulier (2011b), l'ethos de certains milieux professionnels est susceptible de ne pas favoriser la conscience des "possibles", notamment en matière de droits sociaux « ne fut-ce que par la conscience des dispositifs, la valence accordée à leur utilisation et la perméabilité aux remarques des pairs ». La majorité des femmes cadres interrogées exercent dans des environnements masculins. Elles expriment finalement un certain isolement sur ces questions, en partie lié à leur statut. recherche.

En résumé, le tableau n ${ }^{\circ}$ apporte une vision comparée des principaux résultats de notre

Tableau 2. Approche synoptique des résultats

\begin{tabular}{|l|l|}
\hline \multicolumn{1}{|c|}{ Femmes chefs d'entreprise } & \multicolumn{1}{|c|}{ Femmes cadres en PME } \\
\hline 1-La création d'entreprise est perçue & 1- La PME est vécue comme un véritable choix \\
comme un enfantement. Utilisation du \\
registre du discours de la famille pour \\
parler de l'entreprise.
\end{tabular} $\begin{aligned} & \text { polyvalence et de progression. Les aspirations } \\
& \text { vers plus de responsabilités, voire }\end{aligned}$

\footnotetext{
${ }^{1}$ Une partie des $23 \%$ ne peut percevoir d'indemnités car ces dirigeantes n'ont pas les conditions requises au regard du droit.
} 


\begin{tabular}{|c|c|}
\hline & entrepreneuriales, sont fortes. \\
\hline $\begin{array}{l}\text { 2-L'entreprise vécue comme une } \\
\text { opportunité d'articuler maternité et vie } \\
\text { professionnelle. }\end{array}$ & $\begin{array}{l}\text { 2- Parallèlement à une volonté de «toujours } \\
\text { progresser professionnellement», la PME pose } \\
\text { des contraintes en termes de présence, d'objectifs } \\
\text { et de subordination qui font que l'articulation } \\
\text { travail-famille se fait en faveur du travail et } \\
\text { pousse au report du } 1^{\text {er }} \text { enfant. }\end{array}$ \\
\hline $\begin{array}{l}\text { 3-Les entrepreneures insistent sur les } \\
\text { différentes formes de soutien du conjoint } \\
\text { en vue d'articuler maternité et travail. }\end{array}$ & $\begin{array}{l}\text { 3- Le conjoint laisse décider la femme cadre : } \\
\text { - cas majoritaire de conjoint cadre, la cadre } \\
\text { «s'autorise » rarement un congé parental, } \\
\text { - cas minoritaire de conjoint non-cadre, le conjoint } \\
\text { se dit prêt à prendre un congé parental pour que la } \\
\text { cadre n'arrête pas sa carrière } \\
\text {-exception: congé utilisé pour reconversion } \\
\text { professionnelle }\end{array}$ \\
\hline $\begin{array}{l}\text { 4- La maternité n'est pas perçue comme } \\
\text { un frein aux objectifs professionnels. Pas } \\
\text { d'information sur une révision à la baisse } \\
\text { de ces objectifs. }\end{array}$ & $\begin{array}{l}\text { 4-Tout congé lié à la maternité est perçu comme } \\
\text { un «frein à la carrière », néanmoins une structure } \\
\text { avec masse salariale stable semble faciliter la } \\
\text { prise de congé parental à temps partiel. Maternité } \\
\text { perçue comme un frein du développement de } \\
\text { carrière dans les structures où la masse salariale } \\
\text { est en hausse et où s'ouvrent des opportunités } \\
\text { d'évolution. }\end{array}$ \\
\hline $\begin{array}{l}\text { 5- Connaissance et usage (aménagé) des } \\
\text { droits au congé de maternité et activité } \\
\text { souvent ininterrompue pendant la } \\
\text { maternité }\end{array}$ & $\begin{array}{l}\text { 5- Ethos cadre facteur d'une connaissance floue et } \\
\text { limitée des droits à congé maternité/parental. }\end{array}$ \\
\hline
\end{tabular}

\section{Conclusion, limites et perspectives de la recherche}

L'objectif central de notre étude était d'apporter un éclairage empirique et comparatif sur les représentations et le vécu de la maternité, recueillis auprès de deux groupes de femmes au statut différent, des dirigeantes et des cadres dans de petites structures en croissance. Au terme de notre recherche, il apparait qu'être à son compte ou être soumise à un lien de subordination n'a pas les mêmes conséquences sur la vision que ces femmes disent avoir de la maternité. Clairement, la maternité est présentée comme un levier dans la vie professionnelle des entrepreneures et, au contraire, un frein à leur carrière aux yeux des cadres de PME en croissance.

En termes d'application managériale, il convient donc de s'interroger sur l'opportunité qu'auraient les PME en croissance de mettre en œuvre une politique prenant en compte la maternité et ses contraintes, avec des aménagements, comme le télétravail (Saint-Onge, Haines et Sevin, 2000), des facilités de garde d'enfants ainsi qu'un meilleur soutien organisationnel pour permettre aux cadres de mieux concilier emploi et famille (Grodent, Tremblay, 2013). De telles pratiques managériales pourraient être un vecteur de motivation, de fidélisation, voire de recrutement de cadres de talent.

Sur le plan théorique, notre recherche met en lumière l'importance des éléments de contextualisation qui permettraient d'enrichir et d'affiner les modèles théoriques actuels sur les liens travail-famille (Edwards et Rothbard, 2000; Jennings et McDougald, 2007). Nous soulignons le rôle possible de la variable statut professionnel, absente des modélisations des 
liens complexes entre sphères professionnelle et privée. L'ethos cadre attaché au statut cadre dépasse les frontières sectorielles. L'impact de la taille de l'entreprise doit aussi être analysé.

Notre contribution relativise l'idée répandue que les femmes ne créent leur entreprise que lorsque leurs enfants sont en âge de fréquenter l'école (Carrier, Julien et Menvielle, 2006). En effet, notre recherche démontre que les entrepreneures s'appuient sur leur entourage et bénéficient d'une organisation de vie leur permettant un investissement professionnel continu pendant la maternité. Les jeunes mampreneurs de notre échantillon se disent satisfaites de leur équilibre entre sphères professionnelle et privé, tout en maintenant la croissance, ce qui rejoint les travaux de Léger-Jarniou (2013) et Shelton (2006).

Par ailleurs, l'interprétation de nos résultats empiriques doit tenir compte d'un certain nombre de limites méthodologiques. Tout d'abord, une analyse plus poussée permettrait de faire ressortir les similitudes et les différences au sein de chaque groupe (des dirigeantes et des cadres) en fonction de la taille de l'entreprise et du secteur d'activité. Ensuite, la portée de notre étude est limitée par sa nature exploratoire: les résultats issus des vingt-quatre jeunes interlocutrices en France ne sont pas généralisables. En outre, le protocole d'entretien ne pouvait permettre que d'évaluer la représentation que ces femmes ont des dispositifs de congés liés à la maternité, la légitimité accordée à l'utilisation de ces derniers et la façon dont elles se projettent dans l'usage de leurs droits. Aussi, seule une observation prolongée des terrains permettrait de savoir si les représentations qu'elles ont ainsi données d'elles-mêmes dans les interviews sont en accord ou non avec leurs pratiques.

Il conviendrait donc d'ouvrir de nouveaux axes de recherche sur la contextualisation des liens travail-famille. Tout d'abord, il faudrait comparer nos données avec celles que l'on pourrait recueillir auprès de femmes entrepreneures par nécessité ou célibataires ayant à charge un ou des enfants car il est à prévoir que leur représentation de l'entreprise et de la maternité diffère de la représentation des dirigeantes interrogées. Ensuite, des études d'approfondissement sur les mécanismes d'action des variables contextuelles nous semblent prometteuses : la position sociale occupée joue-t-elle un rôle déterminant sur le locus of control du processus de répartition entre temps de travail et temps familial ? Peut-on plus finement évaluer les représentations de l'impact de la maternité sur la vie professionnelle en distinguant entre différents types de croissance (chiffre d'affaires, effectifs...) ? .

\section{Bibliographie}

APEC (2011), Enquête Situation professionnelle et rémunération des cadres, », consulté le 29/12/2014, http://cadres.apec.fr/Emploi/Marche-Emploi/Les-Etudes-Apec APCE (AGENCE POUR LA CREATION D'ENTREPRISE) et OPINIONWAY (2012), Les femmes et la création d'entreprise, consulté le 29/08/2014 http://www.opinionway.com/pdf/sondage_les_femmes_et_la_creation_d_entreprise__sde_paris_2012_pour_impressionx.pdf

APCE (2007), L'entrepreneuriat féminin dans les PME et TPE françaises.

BARNETT R., HYDE J. (2001), «Women, men, work and family : an expansionist theory”, American psychologist, vol.56 (10), p.781-796. 
BARNETT R., RIVERS C. (1998), How two income families are happy, healthy and striving, Mass. Harvard Univ. Press.

BARRERE-MAURISSON M-A. (2003), Travail, famille : le nouveau contrat, coll. Le Monde actuel Gallimard.

BARRERE-MAURISSON M-A. (1992), La division familiale du travail. La vie en double, PUF.

BAH T. (2009), « La transition cédant-repreneur : une approche par la théorie du deuil », Revue Française de Gestion, mai, p 123-148.

BATTAGLIOLA F. (2009), La loi Engerand, première loi sur la protection de la maternité, http://www.archivesdefrance.culture.gouv.fr/action-culturelle/celebrations-nationales/2009/viepolitique/loi-engerand.

BELLE R., NOVELLA S. (2010), «Les services à la personne», INSEE n 140, février.

BLANCHARD S. (2009), "Les femmes cadres et dirigeantes d'entreprise en Europe», CNAFInformations sociales, $\mathrm{v} .1, \mathrm{n}^{\circ} 151, \mathrm{p} .72-81$.

BOSMA N., LEVIE J. (2009), "Global Entrepreneurship Monitor”, Babson College, USA.

BOUTILLIER S. (2008), «Femmes entrepreneures : motivations et mobilisation des réseaux sociaux », Humanisme et entreprise, $\mathrm{n}^{\circ} 290, \mathrm{p} 21-38$.

BOYER B. (2004), «Les pères bénéficiaires de l'APE», Recherche et prévisions, $\mathrm{n}^{\circ}$ 76, p.53-62. BANQUE PUBLIQUE D’INVESTISSEMENT (BPI) (2014), « la création au féminin », dossier Les femmes dans l'économie, consulté le 29/12/2014, http://www.bpifrance-lelab.fr

BREWSTER K., RINDFUSS R. (2000), «Fertility and women's employment in industrialized nations », Annual Review of Sociology, n 26, p.271-296.

BRUSH C. G. (1992), "Research On Women Business Owners", Entrepreneurship Theory And Practice, vol.16 (4), p.5-30.

CAISSE D’ÉPARGNE (2012), Premier baromètre des femmes entrepreneures, consulté le 29/12/2014, http://www.entreprendre-ensemble.com/Brochurebarometre.pdf

CARRIER C., JULIEN P.A. et MENVIELLE W. (2006), «Un regard critique sur l'entrepreneuriat féminin», Revue Gestion HEC Montréal, vol. 31, p.36-50.

CETTE G., DROMEL N., MEDA D. (2005), «Conciliation entre vies professionnelle et familiale et renoncement à l'enfant », Revue de l'OFCE, ${ }^{\circ} 92$.

CHAUDAT P. (2007), "Une réforme organisationnelle sans précédent : le régime social des travailleurs indépendants », Les cahiers de prévoyance et de retraite de la $S N C F, \mathrm{n}^{\circ} 11$, p.37-48. CHAUVEL L. (2010), Le destin des générations structure sociale et cohortes en France du XXe siècle aux années 2010, PUF.

CHENEVIER L. (1996), Les variables influençant l'ampleur du conflit "emploi-famille » ressenti par l'employé(e), Thesis (Ms), Université de Montréal, EHEC, $172 \mathrm{p}$.

CLAIN S. (2000), "Gender differences in full-time self-employment", Journal of Economics and Business, 52, p.245-248.

CORNET A, CONSTANTINIDIS C. (2004), «Entreprendre au féminin : une réalité multiple et des attentes différenciées », Revue Française de Gestion, no 151, p 191204.

D'ANDRIA A., GABARRET I. (2014), «Les mampreneurs françaises : des créatrices d'entreprise à contre-courant ? », XIIème Congrès CIFPME, Agadir.

DAVIDSON P., HONIG B. (2003). "The Role of Social and Human Capital among Nascent Entrepreneurs", Journal of Business Venturing, vol.18, p.301-331.

DAVIE E. (2012), « Premier enfant à 28 ans » Insee première ${ }^{\circ} 1419$.

DUBERLEY J., CARRIGAN M. (2013), “The career identities of 'mumpreneurs' : women's experiences of combining enterprise and motherhood', International Small Business Journal, March, vol.31 (6), p.629-651.

EDWARDS J.R., ROTHBARD N.P. (2000), "Mechanisms linking work and family ", Academy of Management Review, vol. 25 (1), pp.178-199.

ENTENTE DES GENERATIONS POUR L'EMPLOI ET L'ENTREPRISE (EGEE) (2008), «Femmes entrepreneures », consulté le 1//2015 http://www.egee.asso.fr/IMG/pdf/femmes.pdf 
ESPING-ANDERSEN G. (2009), The incomplete revolution: adapting to women's new roles, Polity Press, Cambridge.

EZZEDEEN S., RITCHEY K. (2008), "The man behind the woman: A qualitative study of the spousal support received and valued by executive women", Journal of Family Issues, 29(9), 1107-1135.

FAMILIES AND WORK INSTITUTE (1998), Business Work-Life Study, http://www.familiesandwork.org

FITZGERALD M.A., WINTER M. (2001), «The intrusiveness of homebased work on family life », Journal of Family and Economic Issues, vol. 22 (2), p.75-92.

FUSULIER B. (2011a), Articuler vie professionnelle et vie familiale, étude de trois groupes professionnels, P.U. de Louvain.

FUSULIER B. (2011b), «Le concept d'ethos», Recherches sociologiques et anthropologiques, n०42-2, p.97-109.

FUSULIER B., LALOY D., SANCHEZ E. (2007), «L'acceptabilité sociale de l'usage de congés légaux pour raisons parentales : le point de vue des cadres d'une grande entreprise », Recherches sociologiques et anthropologiques, $\mathrm{n}^{\circ} 38-2$ p.83-103.

GREENHAUS J., POWELL G. (2006), « When work and family are allies: A theory of workfamily enrichment », Academy of Management Review, 31(4), p.72-92.

GREENHAUS J., BEUTELL N. (1985), "Sources of conflict between work and family roles", Academy of Management Review, 10, p.76-88.

GRODENT F., TREMBLAY D.G. (2013), «La conciliation vie privée-vie professionnelle des gestionnaires hommes et femmes : le cas d'une société de transport québécoise »@GRH n ${ }^{\circ} 6$ p.117 à 147.

GRZYWACZ J., MARKS N. (2000), « Reconceptualizing the work-family interface », Journal of Occupational Health Psychology, vol. 5, p.111-126.

GOLDIN C. (2006), "The Quiet Revolution That Transformed Women's Employment, Education, And Family », American Economic Review, v.96, n², p.1-21.

HARRIS C., MORRISON R., HO M., LEWIS K. (2008), "Mumpreneurs: Mothers in the business of babies", 22nd Annual Australian Academy of Management Conference, Auckland, New Zealand.

HAKIM C. (2000), Work-Lifestyle Choices in the 21st Century, OUP.

JANSSEN F. (2005), «La conceptualisation de la croissance», Gestion 2000, nov.-déc., p.267291.

JENNINGS J., MCDOUGALD M. (2007), "work-family interface experiences and coping strategies: implications for entrepreneurship research and practice", Academy of Management Review, vol. 32 (3), p.747-760.

KANTER R. M. (1977), Work and family in the United States: a critical review and agenda for research and policy, Russell Sage Found..

KIRKWOOD J., TOOTELL, B. (2008), "Is Entrepreneurship The Answer To Achieving WorkFamily Balance?", Journal of Management and Organization, vol. 14 (3), pp. 285-302.

LA HALDE (2011), Rapport annuel, Paris.

LEGER-JARNIOU C. (2013), «Femmes entrepreneurs et forte croissance: est-ce possible ?» 8eme congrès de l'Académie de l'entrepreneuriat et de l'innovation, HEG-Fribourg.

LAMBRECHT J., PIRNAY F., AMEDODJI P., AOUNI Z. (2003), Entrepreneuriat féminin en Wallonie, Centre de Recherche PME et d'Entrepreneuriat, EHSAL, 231 p.

LOSCOCCO K., LEICHT K (1993), " Gender, work-family linkages, and economic success among small business owners », Journal of Marriage and the Family, vol. 55, n4, 1993, p.875887.

MARTIN C. (2010), «Concilier vie familiale et vie professionnelle : un objectif européen dans le modèle français des politiques de la famille ? », CNAF informations sociales, $\mathrm{n}^{\circ} 157$, p.114123.

MATTIS M.C., (2000), «Women entrepreneurs in the United Sates», DAVIDSON M.J., BURKE R.J. (dir.), Women in Management: Current Research Issues, Sage, p. 53-68. 
MEDA D. (2009), «Les conditions d'une paternité active», C. NICOLE-DRANCOURT, Conciliation travail-famille: attention travaux, L'Harmattan, p.181-188.

NIKINA A., LE LOARNE S, SHELTON L.M. (2012), « Le rôle de la relation de couple et du soutien du conjoint dans l'entrepreneuriat féminin », Revue de l'Entrepreneuriat, vol. 11 (4), p.37-60.

NAKARA W., FAYOLLE A., «Les «bad » pratiques d'accompagnement à la création d'entreprise » le cas des Entrepreneurs par necessité, in Revue Française de Gestion, 2012/9-10 $n^{\circ}$ 228-229, p. 231-251.

PARASURAMAN S., PUROHIT Y., GODSHALK V., BEUTELL N. (1996), « Work and family variables, entrepreneurial career success, and psychological well-being», Journal of Vocational Behavior, vol. 48 (2), p.275-300.

PAILOT P. (2012), « Mon entreprise, mon amour... de la difficulté des dirigeants à transmettre leur entreprise », Colloque "Les enjeux de la transmission d'entreprise 》, Faculté d'Administration et de Gestion, Montpellier, 5-6 déc., p. 176-188.

POINT S.,RETOUR D. (2009), « Méthodes émergentes et thématiques de Recherche en gestion des Ressources Humaines». XXème Congrès de l'AGRH,Toulouse.

PRETOT X. (2011), Droit de la sécurité sociale, Dalloz.

RATTE S. (1999), «Les femmes entrepreneures au Québec: qu'en est-il ?», Fédération canadienne de l'entreprise indépendante, $18 \mathrm{p}$.

RICHOMME-HUET K. , D'ANDRIA A. (2013), «L'accompagnement entrepreneurial par et pour les mampreneurs », Management International,p 100-157.

ROTHBARD N. (2001), «Enriching or Depleting ? The Dynamics of Engagement in Work and Family Roles », Administrative Science Quarterly, v.46, n4, p.655-684.

ROUSSEAU D. M. (1995), Psychological Contracts in Organizations, Sage.

RSI (2012), Enquête prestations maternité pour les femmes chefs d'entreprise, Etude santé.

RSI Auvergne (2013), extraction des demandes d'indemnisation maternité de chefs d'entreprise de la région Auvergne pour l'année 2012.

SAINT-ONGE S., HAINES V., SEVIN H. (2000), «L'influence de l'appui du supérieur hiérarchique, des collègues et des dirigeants d'entreprise sur le succès en télétravail », Relations Industrielles, $\mathrm{n}^{\circ} 55$ (3), $\mathrm{p} 414-450$.

SAINT-ONGE S., RENAUD S., GUERIN G., CAUSSIGNAC E. (2002), « Vérification d'un modèle structurel à l'égard du conflit travail-famille », Industrial Relations, vol. 57 (3), p.491516.

SCHINDEHUTTE M., MORRIS M., BRENNAN, C. (2003), «Entrepreneurs and motherhood: Impacts on their children in South Africa and the United States», Journal of Small Business Management, vol.41 (1), p. 94-107.

SHELTON L.(2006), "Female Entrepreneurs, Work-Family Conflict, and Venture Performance", Journal of Small Business Management, vol. 44 (2), pp. 285-297.

SILVERA R. (2002), Articuler vie familiale et vie professionnelle en Europe, La Documentation française.

SMAILI N. (2002), «Les femmes employeurs du Québec : quelques statistiques », Cahier de recherche. HEC Montréal.

SUMER H. C, KNIGHT P. (2001), "How do people with different attachment styles balance work and family?", Journal of Applied Psychology, n86, p. 653-663.

THIETART R.A. (2006), Méthodes de recherche en management (3e éd.), Dunod, Paris.

TORRES O., (2007), «La recherche académique française en $P M E »$, La Documentation Française - Oséo, $136 \mathrm{p}$.

TREMBLAY D.-G., LARIVIERE M. (2009), «La conciliation emploi-famille dans le secteur de la santé au Québec», Genre et diversité en gestion, Vuibert, p.101-118.

TREMBLAY D.-G. (2012), Conciliation emploi-famille et temps sociaux, Presses de l'Université du Québec.

VANDERSCHELDEN M. (2006), «Position sociale et choix du conjoint», INSEE. 
ZARCA B., (2009), «L'ethos professionnel des mathématiciens", Revue française de sociologie, vol.50 (2), p.351-384. 


\section{Annexes :}

Données sociodémographiques des dirigeantes. Pour des raisons de confidentialité tous les prénoms sont fictifs.

\begin{tabular}{|c|c|c|c|c|c|c|c|c|c|}
\hline Prénom & Age & Entreprise & $\begin{array}{l}\text { Situat } \\
\text { géogr. }\end{array}$ & Ancienneté & Secteur & Activité & $\begin{array}{l}\text { Situation } \\
\text { conjoint }\end{array}$ & $\begin{array}{l}\text { Nombre } \\
\text { de } \\
\text { salariés }\end{array}$ & $\begin{array}{l}\text { Nombre } \\
\text { d'enfants }\end{array}$ \\
\hline Audrey & 35 & SAS & 83 & 5 & $\begin{array}{c}\mathrm{E} \\
\text { business }\end{array}$ & $\begin{array}{c}\text { Comparateurs et } \\
\text { moteurs de } \\
\text { shopping spécialisés }\end{array}$ & Entrepreneur & sarans & 1 \\
\hline Jessica & 29 & SARL & $\begin{array}{c}14 \\
75,44\end{array}$ & 4 & $\begin{array}{c}\mathrm{E} \\
\text { business }\end{array}$ & $\begin{array}{c}\text { Gestionnaire de } \\
\text { sites internet et } \\
\text { boutique physique }\end{array}$ & Cadre & 0 & 2 \\
\hline Cécile & 34 & SARL & 74 & 6 & $\begin{array}{c}\mathrm{E} \\
\text { business }\end{array}$ & $\begin{array}{c}\text { Pure player, vente } \\
\text { de mobilier }\end{array}$ & Cadre & 0 & 2 \\
\hline Marion & 32 & SARL & 33 & 3 & $\begin{array}{l}\text { Service à } \\
\text { la } \\
\text { personne }\end{array}$ & $\begin{array}{c}\text { Dédié } \\
\text { exclusivement aux } \\
\text { personnes en } \\
\text { situation de } \\
\text { handicap }\end{array}$ & Cadre & 1 & 1 \\
\hline Aurélie & 31 & SARL & 75 & 6 & $\begin{array}{l}\text { Service à } \\
\text { la } \\
\text { personne }\end{array}$ & Evénementiel & Cadre & 4 & 2 \\
\hline Chloé & 35 & SARL & 38 & 3 & $\begin{array}{l}\text { Service à } \\
\text { la } \\
\text { personne }\end{array}$ & $\begin{array}{c}\text { Micro crèche : } \\
\text { accueil, éveil et } \\
\text { garde de jeunes } \\
\text { enfants de } 0 \text { à } 3 \text { ans }\end{array}$ & Employé & 3 & 4 \\
\hline Jeanne & 35 & SARL & 44 & 8 & $\begin{array}{l}\text { Service à } \\
\text { la } \\
\text { personne }\end{array}$ & $\begin{array}{l}\text { Service à domicile } \\
\text { (ménage/repassage } \\
\text { et garde d'enfants) }\end{array}$ & Cadre & 1 & 2 \\
\hline Leila & 32 & SAS & 34 & 4 & $\begin{array}{c}\text { Service à } \\
\text { la } \\
\text { personne }\end{array}$ & $\begin{array}{l}\text { Garde d'enfants à } \\
\text { domicile }\end{array}$ & Cadre & 250 & 1 \\
\hline Charlotte & 35 & SARL & 36 & 9 & $\begin{array}{l}\text { Service à } \\
\text { la } \\
\text { personne }\end{array}$ & $\begin{array}{l}\text { Service à domicile } \\
\text { (ménage/repassage } \\
\text { et garde d'enfants) }\end{array}$ & Cadre & 8 & 4 \\
\hline Pauline & 35 & SARL & 92 & 5 & $\begin{array}{l}\text { Service à } \\
\text { la } \\
\text { personne }\end{array}$ & $\begin{array}{l}\text { Animation pour } \\
\text { enfants }\end{array}$ & Cadre & 2 & 3 \\
\hline Elodie & 35 & SARL & 75 & 6 & $\begin{array}{l}\text { Service à } \\
\text { la } \\
\text { personne }\end{array}$ & $\begin{array}{l}\text { Service à domicile } \\
\text { (ménage/repassage } \\
\text { et garde d'enfants) }\end{array}$ & Cadre & 3 & 1 \\
\hline Sonia & 31 & SARL & 75 & 3 & $\begin{array}{l}\text { Service à } \\
\text { la } \\
\text { personne }\end{array}$ & $\begin{array}{l}\text { Service à domicile } \\
\text { (ménage/repassage } \\
\text { et garde d'enfants) }\end{array}$ & Employé & 2 & 3 \\
\hline
\end{tabular}

Données sociodémographiques des femmes cadres en PME. Pour des raisons de confidentialité tous les prénoms sont fictifs.

\begin{tabular}{|l|l|l|l|l|l|l|c|c|}
\hline Prénom & Age & Fonction & Ancienneté & $\begin{array}{l}\text { Situation } \\
\text { géo. }\end{array}$ & Activité & $\begin{array}{l}\text { Situation } \\
\text { conjoint }\end{array}$ & $\begin{array}{c}\text { Nombre } \\
\text { de } \\
\text { salariés }\end{array}$ & $\begin{array}{c}\text { Nombre } \\
\text { d'enfants }\end{array}$ \\
\hline Sarah & 27 & Chef de & 2 ans & 75 & Luxe & Cadre expert- & 120 & 1 \\
\hline
\end{tabular}




\begin{tabular}{|c|c|c|c|c|c|c|c|c|}
\hline & & $\begin{array}{l}\text { produit- } \\
\text { Acheteur }\end{array}$ & & & & comptable & & \\
\hline Marie & 32 & $\begin{array}{l}\text { Consultante } \\
\text { communication }\end{array}$ & 7 ans & 75 & $\begin{array}{l}\text { Conseil } \\
\text { patrimoine }\end{array}$ & Cadre BTP & 12 & 0 \\
\hline Lucie & 33 & Consultante & 7 ans & 93 & $\begin{array}{l}\text { Conseil } \\
\text { logis. }\end{array}$ & Technicien & 9 & 0 \\
\hline Justine & 26 & Consultante & $\begin{array}{c}2 \text { ans, } 3 \\
\text { mois }\end{array}$ & 40 & Conseil SI & Cadre & 220 & 0 \\
\hline Carine & 30 & $\begin{array}{l}\text { Ingénieur } \\
\text { commercial }\end{array}$ & 2 ans & 74 & $\begin{array}{l}\text { Editeur } \\
\text { Logiciel }\end{array}$ & Architecte & 200 & 1 \\
\hline Rose & 35 & $\begin{array}{l}\text { Assistante } \\
\text { direction }\end{array}$ & 8 ans & 34 & Immobilier & $\begin{array}{l}\text { Ingénieur } \\
\text { comm }\end{array}$ & 1 & 2 \\
\hline Lise & 29 & $\begin{array}{l}\text { Chargée du dvt } \\
\text { commercial et } \\
\text { marketing }\end{array}$ & 2 ans & 69 & $\begin{array}{l}\text { Conseil et } \\
\text { prospection }\end{array}$ & Non cadre & 4 & 0 \\
\hline Léa & 33 & $\begin{array}{l}\text { Responsable } \\
\text { admin. et } \\
\text { financier }\end{array}$ & 7 ans & 34 & $\begin{array}{l}\text { Conseil } \\
\text { (techno. } \\
\text { vinicole) }\end{array}$ & $\begin{array}{l}\text { Non-cadre } \\
\text { Placement } \\
\text { Assurances-vie }\end{array}$ & 50 & 0 \\
\hline Anne & 32 & Resp. marché & 3 ans & 63 & Internet & Dirigeant PME & 35 & 1 \\
\hline Zoé & 29 & $\begin{array}{l}\text { Resp. } \\
\text { communicat. }\end{array}$ & 2 ans & 34 & $\begin{array}{l}\text { Cosmétiques } \\
\text { bio }\end{array}$ & Non-cadre & 5 & 0 \\
\hline Cindy & 30 & $\begin{array}{l}\text { Resp. } \\
\text { recrutement } \\
\text { (resp. élargies) }\end{array}$ & 5 ans & 34 & SSII & $\begin{array}{l}\text { Cadre analyste } \\
\text { financier } \mathrm{GE}\end{array}$ & 130 & 1 \\
\hline Pascale & 30 & RRH & 6 ans & 63 & Plasturgie & Cadre GE & 240 & 0 \\
\hline
\end{tabular}

